\title{
Job Scheduling Using Fuzzy Neural Network Algorithm in Cloud Environment
}

\author{
V. Venkatesa Kumar and K. Dinesh
}

\begin{abstract}
Cloud Computing is providing computing as a service rather than product such as shared resources, software information, etc...Cloud computing can be used for dispatching user tasks or jobs to the available system resource like storage and software. Scheduling algorithm is used for dispatching user tasks. In Job scheduling using fuzzy neural network algorithm, first user tasks are classified based on Quality of service parameters like bandwidth, memory, CPU utilization and size. The classified tasks are given to fuzzier where the input values are converted into the range between 0 and 1 . Neural network contains input layer, hidden layer and output layer for adjusting the weight of user task and match with system resources. The function of de-fuzzier is to reverse the operation performed by fuzzier. The exemplar input is matched with the exemplar output label by adjusting weights. The algorithm is implemented with the help of simulation tool (CloudSim) and the result obtained reduces the total turnaround time and also increase the performance.
\end{abstract}

Keywords--- Cloud Computing, Neural Network, Fuzzy Logic, Job Scheduling, Berger Model

\section{INTRODUCTION}

$\mathrm{S}$ CHEDULING is used to allocate particular resources for a certain tasks in particular time[9]. Job scheduling problem is a core and challenging issue in cloud computing. The job execution time cannot be predicted in cloud computing. Hence the scheduler must be dynamic. The purpose of scheduling is to increase the utilization of resources. The cloud computing is a large group of interconnected computers and cloud symbol represents a group of systems or complicated networks. Cloud computing is one way of communication among the various system in the network with the help of internet.

Cloud computing is "on demand resources provisioning" which means to provide the available resources based on the requirement of the resources. Cloud computing contains a central remote server to maintain the data and application. Cloud computing is a "subscription based". Cloud computing is a pay as per usage and reliable leads to an efficient network. Cloud Computing is an emerging technique and it's very successful because of the following features like reliable, secure, fast, fault tolerance and efficient communication etc., among different network.

Cloud computing are typically classified into two types such as types of services offered and location of cloud. The services are broadly classified as Platform as a service (PaaS),

V. Venkatesa Kumar, Assistant Professor, Anna University of Technology, Coimbatore.

K. Dinesh, Anna University of Technology
Infrastructure as a service (IaaS) and Software as a service (SaaS), etc... Based on the location cloud computing can be classified into four types like private cloud, public cloud, hybrid cloud and community cloud. Fig 1 describes the general structure of cloud computing.

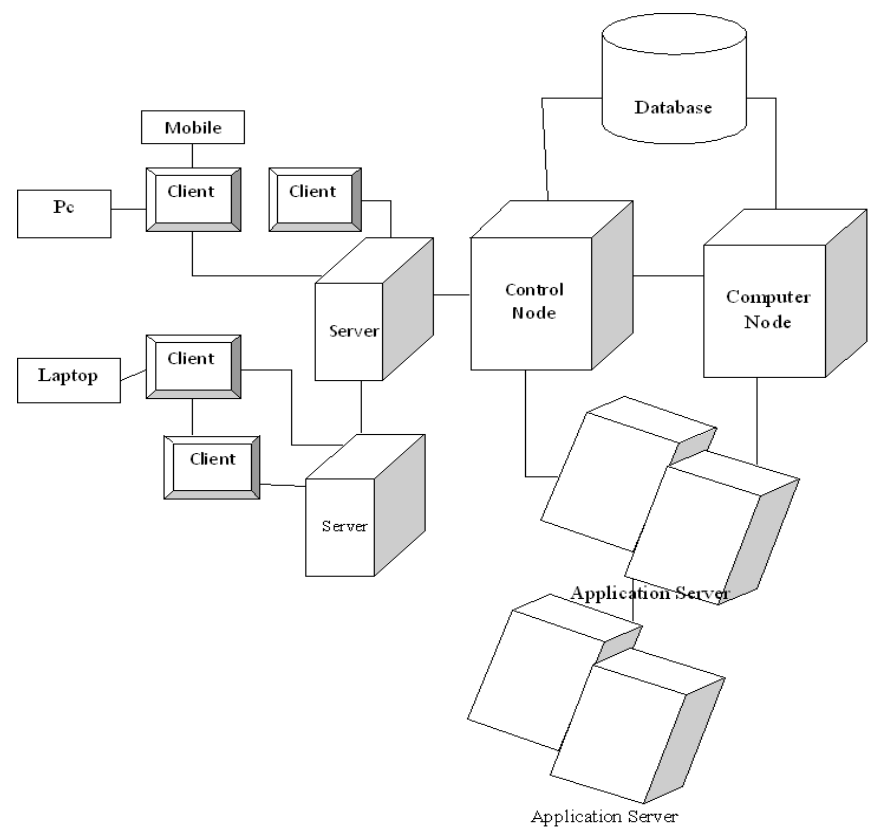

Figure 1: Cloud Computing

Virtualization is the ability to run multiple operating systems on a single physical system and share the underlying hardware resources. One of the fundamental aspects of virtualization technologies employed in Cloud environments is resource consolidation and management. Virtualization is a way to abstract the hardware and system resources from operating system. Hypervisor or Virtual Machine Monitor (VMM) is lies in between the hardware and the Operating System (OS). There are two types of virtualization available like Para virtualization and full virtualization. Normally in cloud computing uses only Para virtualization.

Figure 2 describes the detailed the relation between Fuzzy logic and Neural Network. Neural Network contains three layers like input layer, hidden layer and output layer [11]. The term Neural Network describes the adjusting weights of the hidden layer to match the input and output. No mathematical model is necessary for neural network. It's learning from examples. In neural network initially behaves as block box behavior. In hidden layer alone consists of many layers for adjusting the weight for mapping. In fuzzy logic normally consists of a linguistic variable like high, medium and low [10]. In Fuzzy Neural Network consists of fuzzification which normally converts all the input values into the range between 0 
and 1.The de-fuzzification performs reverse operation of fuzzification [7].

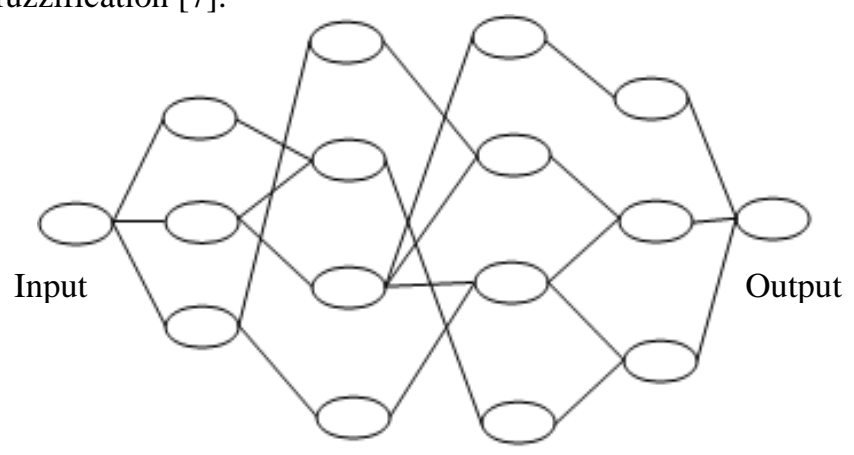

Hidden layers

Figure 2: Fuzzy Neural Network

\section{LITERATURE SURVEY}

The user tasks are classified based on the QoS parameters like size, bandwidth and expectation time. The task can be mapped with the system resources and if matching occurs then the task is completed within the expectation time. Otherwise, adjust the general expectation and then map with the system resources. Suppose the user task is not mapped with the system resources after adjusting the general expectation, the task remains incomplete [1].

Micro-economic model consists of two principles namely auctioning and commodity market. In commodity approach, the tasks are allocated for individual as well as for bundled resources. The resources are classified into different classes based on the CPU cycles, disk space, network bandwidth and memory space. The price of a particular resource is fixed only after equilibrium has maintained among demand and supply. Suppose both demand increase and supply decrease or vice versa, result reflects in increase price. Once equilibrium is achieved, further price cannot be changed and follow first come first serve $[2,3]$.

The distributed system consists of many resources. The traditional system compare with distributed resources can resemble increase of reliability and performance. The performance of a system is based on two agents like jobs and the processors. Jobs mainly perform three functions namely compute budget set, preference relation and generate most preferred bid. Processors mainly contain the action resources, update price and advertising. This algorithm is similar to consumers and supplier relation. The economics model is used to minimize the complexity of large complex problems into the set of smaller by classify as independent problems [8].

Resources allocation is major role in all computing. In this paper, the authors mainly discussed four approaches. There are State Based (current snapshot), Pre-emptive (migrate to different systems), Non-pre-emptive (execute in host itself) and Model Based (predicts system state). There are three algorithm discussed in this paper such as Round Robin (RR), Proportional Share Protocol (PSP) and Continuous Double Actions (CDA). The parameters considered in the distributed system are message delay, processing delay, task creation, number and speed of the servers. State Based and Non-pre-emptive Based system is to minimize communication overhead of auctions (e.g. divide and conquer).State Based and Pre-emptive are more dynamic and flexible[4].

Popcorn is intermediate between the programmer and a single virtual parallel computer which provides to connect with the internet. It is a globally distributed system. The principle behind Popcorn is market based product. In market terms, selling denotes jobs and buying denotes parallel program in terms of CPU time. The term market is used to connect both jobs and popcorn. There is no direct connection between the sellers and buyers like online trade which needs only minimum requirements. There are two mechanism in Popcorn such as repeated vickrey auction and double auction mechanism[5].

\section{ARCHITECTURE}

In this architecture diagram is mainly describes how the user tasks can be classified and mapped with system resources.

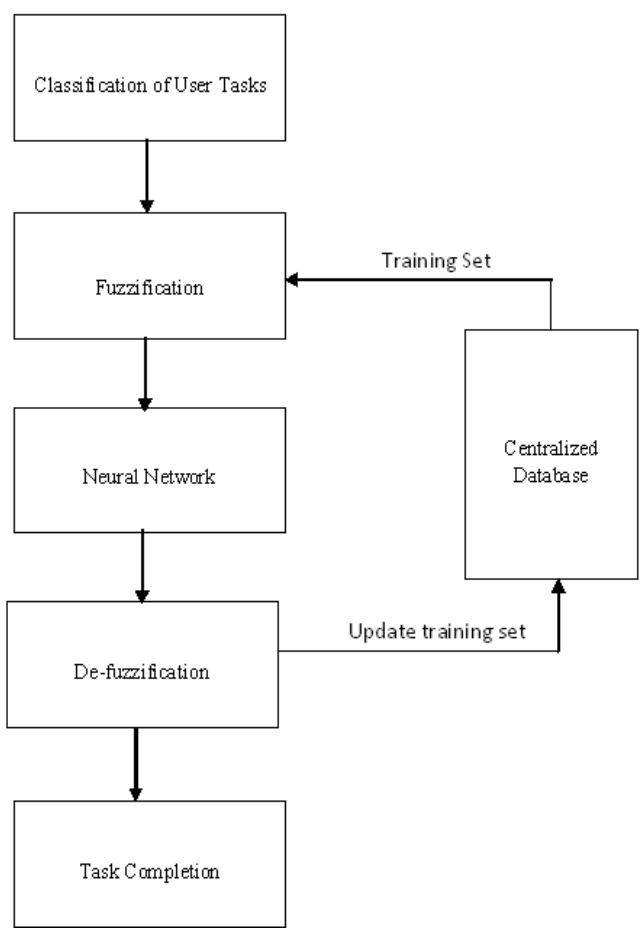

Figure 3: System Architecture

Fig 3 shows that how the user task is mapped with the system resources. The Classification of user tasks is classified based on the parameters like bandwidth, memory, and expected time. The classified inputs are given to the fuzzification where it is used for conversion. The training data in centralized database is taken as an input. The converted input values are passed to the neural network. In neural network will make decision for mapping the system resources with the user tasks. With the help of de-fuzzification, the fuzzy range values are converted into their original values.

In neural network, if the user tasks are not mapped with the system resources and then using back propagation algorithm, the tasks are reclassified and follow the similar operations. The centralized database is used for maintaining and updating the 
training data required for neural networks. The feedback or feed forward algorithm is used to reclassify the user task if is not matched with the system resources.

\section{Methods AND MetRicEs}

\section{A. Berger Model}

In Cloud computing, user task, the resources provider and scheduling system are mainly considered. Particularly in Berger model mainly focuses on job scheduling according to this authors, Baomin Xu, Chunyan Zhao, Enzhao Hu, Bin Hu.

- Task Classification Based on QoS

The QoS (Quality of Services) stems from a list of parameters of Internet performance mechanism. In Berger model, the parameters considered are

- Completion time

- Bandwidth

To measure user satisfaction according to different QoS parameter, different quantification evaluation criteria need to be established for different QoS parameters.

\section{i. $\quad$ Definition 1(Task Justice)}

The actual allocation resources for a task are close to the maximum extent, to the expected resources of the task, called the task to obtain the fair execution. The justice evaluation function as

$$
J_{i}=\theta \ln A R_{i} / E R_{i}
$$

$\theta$ Denotes the constant which varies between $0<\theta \leq 1$. $A R_{i}$ (Actual resources allocation) is the quantity of resources which required for user task actually. Similarly $E R_{i}$ (Expected resources allocation) is a required resource for user task excepted. The value $J_{i}$ is just used to judge the outcome of the resources allocation process.

\section{ii. Definition 2(System Justice)}

Assume the system task set as $\mathrm{T}=\left\{T_{1}, T_{2}, \ldots ., T_{3}\right\}$ and the corresponding system justice as $\mathrm{J}=\left\{J_{1}, J_{2}, \ldots, J_{n}\right\}$. The justice evaluation function for the system is

$$
J=\sum_{i=1}^{n}\left|J_{i}\right|
$$

The whole users achieve maximize fairness when $\mathrm{J}$ achieves the minimum value. The fairness of the entire system is best when compare with actual value.

\section{iii. Definition 3(Integrate General Expectation)}

When the task has multiple expectations preference, integrated general expectation is defined as the mathematical expectation.

$$
\overline{\mathrm{e}}_{\mathrm{ij}}=1 / \mathrm{n} \sum_{\mathrm{j}=1}^{\mathrm{n}} \mathrm{e}_{\mathrm{ij}} \quad(\mathrm{n}=1,2,3)
$$

The bandwidth between two points or nodes can be calculated by using Euclidean distance.

$$
D_{x y}=\sqrt{\left(X_{1}-Y_{1}\right)^{2}+\cdots+\left(X_{n}-Y_{n}\right)^{2}}
$$

\section{iv. $\quad$ Fundamental Concept}

1. Task Model

In a heterogeneous system in a cloud environment, the task system has several features

a. Tasks are aperiodic: The arrival time of each task is not known priori.

b. Tasks are no preemptive: The tasks are independent, which mean it does not any other task for execution.

c. Tasks accesses are mainly divided shared access and exclusive access.

2. System Model

In system model or scheduler is consists of dispatch Queue (DQ). It has been used for collecting all the tasks arrive from different location or different time to a scheduler.

\section{B. Genetic Algorithm}

Genetic algorithm has used for mapping the user tasks with system resources in a fuzzy Neural Network of cloud computing. In genetic algorithm which mainly discuss about encoding, initial population, fitness function, selection. Cross over, mutation and termination of algorithm. In encoding can used to convert the different input values into a specific range. There are different methods available for encoding such as binary encoding, Permutation Encoding, value encoding and tree encoding etc.

Initial population algorithm begins by creating random values. Using initial population, the selection, fitness function, crosses over and mutation are carried out. In Fitness Function is used to check weather this algorithm performed better and produce results within expectation time. It will take major role in genetic algorithm to execute the results. Cross Over is simply a swapping of values present in the initial population and easy to generate new initial values among a pair of chromosome. In Future, these values are used as an initial population. Mutation is similar to cross over but exchanging values within chromosome. In termination process is used to choose best values among a set of population.

\section{Pseudo Code of Resources Selection Process \\ Begin}

Assign training set for collecting all resources ei for $T_{i}, \mathrm{i}=1$ to $\mathrm{m}$

\{

select $\mathrm{VM}_{\mathrm{i}}$ according to the required tasks $\mathrm{T}_{i}$;

\}

Convert values into linguistic variables $\{/ /$ fuzzification methods

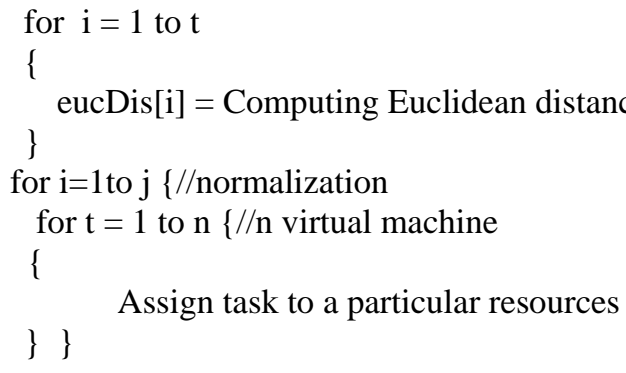




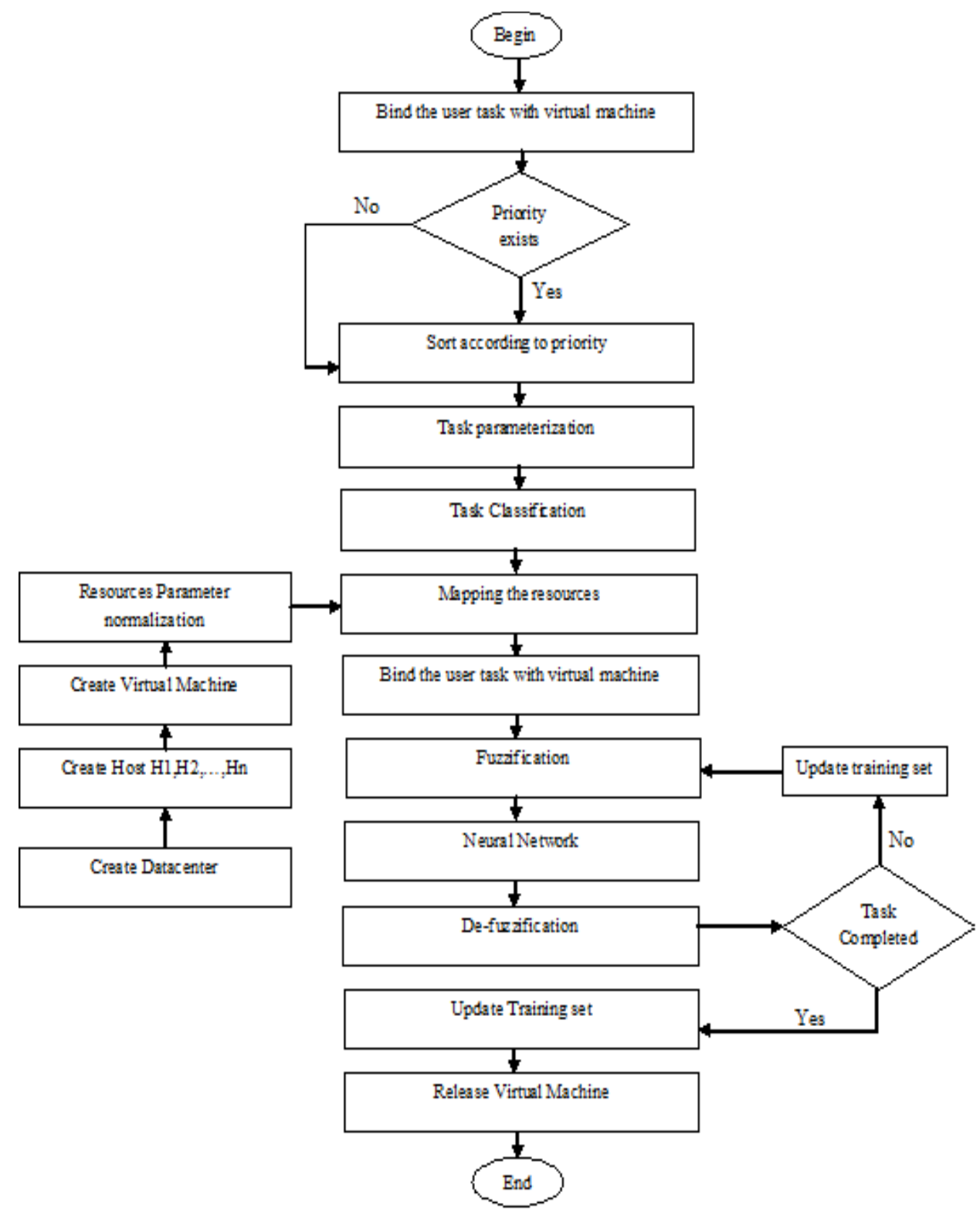

Figure 4: Data Flow Diagram

Update the training set tuple

Select minimum Euclidean distance Do from eucDis[i];

Binding $\mathrm{T}_{i}$ to Do;

Reconvert the linguistic variables into values (// by using defuzzification

End;

\section{Data Flow Diagram}

Fig 4 gives the data Flow Diagram which is used to represent how the resources are allocated to a particular resources using virtual machine. Initially, large number of tasks is submitted to the system. After that, check whether any priorities exist in submitted tasks. If the priority exists, then sort the tasks submitted according to the priority. In task parameterization can be used to check whether all resources are available for particular task to execute. Create a data centre, host and virtual machine is used to avoid heterogeneous environment and resources parameter normalization is to mention all the parameters.

In resources mapping is used to map the resources parameter with user parameters. If the resources are mapped with user task then bind the task with virtual machine. Fuzzification is used to convert the different range of input values into linguistic variables. Genetic algorithm in neural network is used to find out a best result of mapping the user tasks. De-fuzzification is used to convert the linguistic variables into values and check the task has been completed before the expectation time. Suppose, if the task is not mapped with system resources, update the training set and reclassify the user tasks. Otherwise just update the training set and realize the corresponding virtual machine. 


\section{RESUlTS AND DISCUSSION}

\section{A. Simulation Tool}

In Cloud Computing, Simulation can be implemented with the help of CloudSim. In CloudSim, the tasks are to add the scheduling algorithm. The bindCloudletToVM () method in Datacenter Broker class of CloudSim can bind the single task to a designated single virtual machine and run it. To support the search of reasonable virtual machine resources for a particular task, Datacenter Broker class of CloudSim platform need to be extended. Namely, overloading bind Cloudlet ToVM () methods to implement their scheduling algorithm. Cloudlet class is also expectation completion time [6].

\section{B. Experiment Results}

Compare with traditional algorithm of Berger model our proposed work using fuzzy neural network is efficient.

In fig 5 (Comparing Bandwidth utilization) shows that proposed work reduces the bandwidth utilization when compare with traditional approach. Fig 5. is used for calculating the bandwidth utilization.Number of cloudlets is simply represends the number of jobs/tasks submitted to the system. Mainly bandwidth is used for communication among nodes in a network.

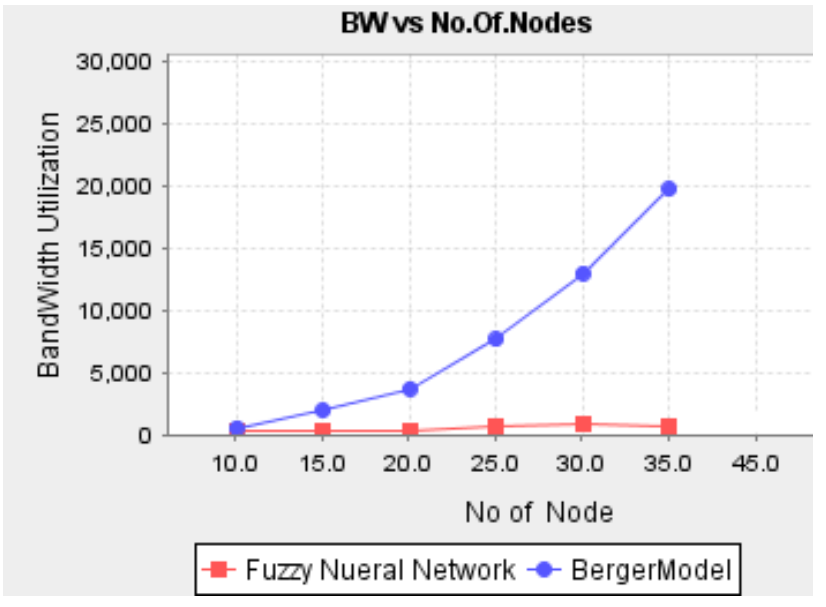

Figure 5: Comparing Bandwidth Utilization

Fig 6 gives the analysis and comparison of performance offered by Berger model with the proposed method of fuzzy neural network model. Here if the number of cloudlets are increased the completion time of the algorithm is decreased linearly when compared with traditional approach. Therefore, the completion time of the proposed method is less than the existing system. Based on the comparison and the results from the experiment show the proposed approach works better than the other existing systems.

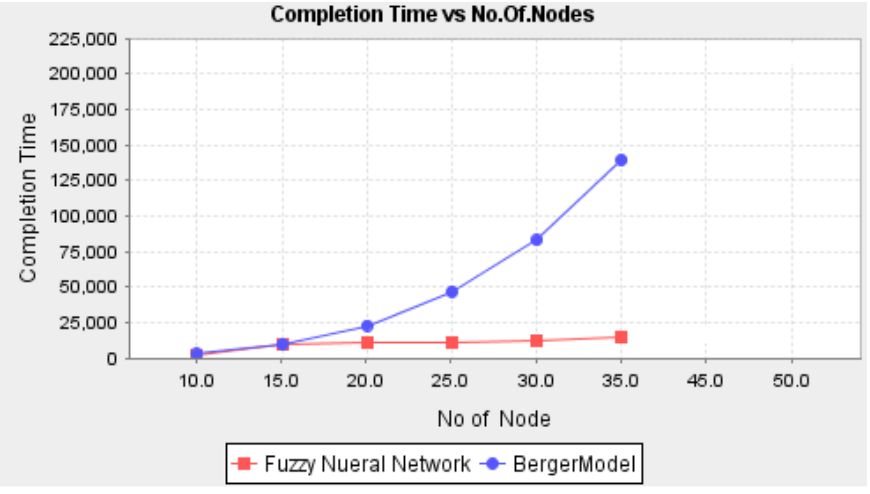

Figure 6: Calculation of Completion Time

\section{CONCLUSION AND FUTURE WORK}

In this paper, the job scheduling using fuzzy neural network reduces the bandwidth utilization, completion time and memory utilization compared than the traditional algorithm. Through CloudSim simulation tool, proposed algorithm is implemented and it shows better performance than the traditional algorithm. Bandwidth, size, exceptation time have been considered as parameters to map the resources. The performance of fuzzy neural network is best for smaller system compare than large system. In future work, the performance of large systems can be increased by using parellel genetic algorihm than genetic algorithm.

\section{REFERENCES}

[1] Baomin Xu a, Chunyan Zhao b, Enzhao $\mathrm{Hu}$ a, Bin $\mathrm{Hu}$ c,d, "Job Scheduling algorithm using Berger model in Cloud Environment", Elsevier in Advances in Engineering Software, Vol. 42 , No. 7, Pp. 419-425, 2011.

[2] Broberg J, Venugopal S, Buyya R., "Market-oriented Grids and utility Computing: the state-of-the-art and future directions", J Grid Computing, Vol. 6, No. 3, Pp. 255-76, 2008.

[3] Ferguson D, Yemini Y, Nikolaou C, “Microeconomic algorithms for load balancing in distributed computer systems", Proceedings of the eighth international conference on distributed systems, San Jose: IEEE Press, Vol. 2, No. 30, Pp. 491-9,1988.

[4] Gomoluch J, Schroeder M., "Market-Based resource allocation for Grid Computing: a model and simulation", In: Endler M, Schmidt D, editors. Proceedings of the first international workshop on middleware for Grid Computing (MGC 2003). Rio de Janeiro: Springer-Verlag, Vol. 6, No. 5, Pp. 211-218, 2009.

[5] Regev O, Nisan N, "The popcorn market - An online market for computational resources", Proceedings of the first international conference on information and computation economies Charleston: ACM Press, Vol. 28, No. 1-2, Pp. 177-189, 2000.

[6] Rajkumar Buyya, Rajiv Ranjan and Rodrigo N. Calheiros, "Modeling and simulation of scalable Cloud Computing environments and the CloudSim Toolkit: challenges and opportunities", Proceedings of the seventh high performance Computing and simulation conference (HPCS 2009, ISBN: 978-1- 4244-49071), Leipzig, Germany. New York, USA: IEEE Press, June 21-24, 2009

[7] B. Gabrys and A. Burgiela, "General fuzzy min-max neural network for clustering and classification", IEEE Trans. Neural Networks, Vol. 11, No. 3, Pp. 769-783, 2000.

[8] Kumaran Subramoniam, Muthucumaru Maheswaran and Michel Toulouse, "Towards a Micro-Economic Model for Resource Allocation in Grid Computing Systems", Electrical and Computer Engineering, IEEE CCECE, Vol. 2, Pp. 782- 785, 2002.

[9] Chenhong Zhao, Shanshan Zhang, Qingfeng Liu, Jain Xie and Jicheng Hu "Independent Tasks Scheduling Based on Genetic Algorithm in Cloud Computing”, IEEE paper 2009, Pp. 978-1-4244-3693-4, 2009.

[10] Carl G.Looney and Sergiu Dascalu, “A Simple Fuzzy Neural Network”, University of Nevada Reno, Vol. 9, No. 2, Pp. 89557, 2009. 
[11] H. M. Lee, C.M. Yen, S.-F. Liang, J.-F. Chung and N.Kumar, "Support vector based fuzzy neural network for pattern classification", IEEE Transaction Neural Networks, Vol. 14, No. 1, Pp. 31-41, 2006.

[12] Rajkumar Buyya, Chee Shin Yeo, Srikumar Venugopal, "Market-oriented Cloud Computing: vision, hype and reality for delivering IT services as computing utilities", the tenth IEEE international conference on high performance Computing and communications (HPCC 2008), Dalian, China. Los Alamitos, CA, USA: IEEE CS Press; September 25-27, 2008.

[13] Braun TD, Siegel HJ, Beck N, "A Comparison of eleven static heuristics for mapping a class of independent tasks onto heterogeneous distributed Computing system", J Parallel Dist Comput, Vol. 61, No. 6, Pp. 810-37, 2001.

[14] LI Mao-Sheng, YANG Shou-Bao, FU Qian-Fei1, YANG Jin, “A Grid Resource Transaction Model Based on Compensation", J Softw , Vol. 17, No. 3, Pp. 472-80, 2006.

[15] Broberg J, Venugopal S, Buyya R, "Market-oriented Grids and utility Computing: the state-of-the-art and future directions", J Grid Comput , Vol 6, No. 3, Pp. 255-76,2008.

[16] Kumar S, Dutta K, Mookerjee V, “ Maximizing business value by optimal assignment of jobs to resources in Grid Computing”, Eur J Oper Res , Vol. 194, No. 3, Pp. 856-72, 2009.

[17] Berger J, Cohen BP, Closer TL, Zelditch M, "Status characteristics and expectation states: a process model", Sociological theories in progress, Boston: Houghton, Vol. 1, Pp. 47-74, 1966.

[18] P.M. Patil and T.R. Sontakke, "Rotation, scale and tanslation invariant handwritten Devanagari numeral character recognition using general fuzzy neural network," Pattern Recognition, Vol. 40, Pp. 2110-2117, 2007.

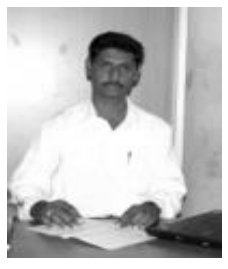

V. Venkatesakumar is currently working as an Assistant Professor in Anna University of Technology Coimbatore. He received Bachelor and Master Degree, B.E and M.E from Anna University Chennai. He has 6 years of Teaching Experience. Currently he is an academician and doing his doctorate. His research area includes Operating System, Software Engineering and Web Technologies.

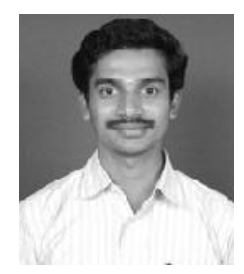

K. Dinesh is pursing M.E in Anna University of Technology. He received Bachelor Degree from Anna University Chennai. His area of interest is Data structure, Database Management System. His research areas are Cloud Computing, Fuzzy Logic and Neural Network. 